\title{
CONF-9.51073--23 \\ A Practical Block Detector for a Depth Encoding PET Camera
}

\author{
Joel G. Rogers*, Christian Moisan*, Emile M. Hoskinson*, \\ Mark S. Andreaco ${ }^{\dagger}$, C. W. Williams ${ }^{\dagger}$, and Ronald Nutt ${ }^{\dagger}$ \\ - TRIUMF, 4004 Wesbrook Mall, Vancouver, B.C., CANADA V65-2A3 \\ ${ }^{\dagger}$ CTI PET Systems, 810 Innovation Drive, Knoxville, TN 37933
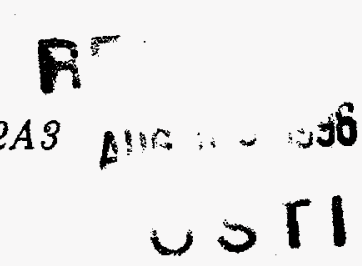

Abstract

The depth-of-interaction effect in block detectors degrades the image resolution in commercial PET cameras and impedes the natural evolution of smaller, less expensive cameras. A method for correcting the measured position of each detected gamma ray by measuring its depth-ofinteraction was tested and found to recover $38 \%$ of the lost resolution in a table-top $50 \mathrm{~cm}$ diameter camera. To obtain the desired depth sensitivity, standard commercial detectors were modified by a simple and practical process, which is suitable for mass production of the detectors. The impact of the detector modifications on central image resolution and on the ability of the camera to correct for object. scatter were also measured.

\section{INTRODUCTION}

The latest commercial PET cameras operate in the volume imaging mode, without interplane septa. Such cameras incorporate $\mathrm{BGO}$ block detectors arranged in a ring around a cylindrical field of view (FOV). The diameter of the ring is an important factor in the cost of the camera, because the number of detectors required is proportional to the diameter of the ring. The smaller the ring, the fewer detectors are required, and therefore the cost of building the camera is lower.

Commercial cameras use a detector ring diameter substantially larger than the FOV diameter. With present commercial detector technology, it is not possible to make the ring diameter much smaller because the image resolution at the edges of the FOV is already limited by the depth-of-interaction (DOI) effect [1]. Gamma ray positions are miscoded by amounts which, on average, increase with the angle that the gamma ray line makes with the long axis of the long narrow crystals which make up the block. As the diameter of the ring is made smaller, gamma rays from the edges of the FOV make larger angles when they enter a block, which worsens the resolution. To avoid excessive loss of resolution, some form of correction for the DOI effect is required in small ring cameras.

A possible solution to the problem of DOI resolution loss was proposed recently by Rogers [2]. This method employs a modified gamma ray detector to evaluate the DOI of each gamma ray detected. When such a measure of DOI is incorporated into an appropriate algorithm [2], the loss of resolution at the edge of the FOV can be partially corrected, giving improved imaging from small-ring cameras. The work described in this article tests practical block detectors designed for implementing the method [2]. Because the new detectors were adapted from stateof-the-art PET detectors, with only minor modifications, the new blocks should cost about the same to manufacture as the conventional blocks, resulting in a significant saving in a small-ring camera due to its smaller number of blocks. The proposed method also offers the possibility of improving the performance of present day cameras in imaging large objects (e.g. the torso), again with little increase in the cost of the individual blocks.

The detectors tested were modified by hand from standard assembly-line detectors intended for the Siemens EXACT HR+ (hrplus) camera. Standard detectors have improved over the years from the original invention of Casey, Nutt, and Douglass [3, 4]. The main improvement. has been an increase in the amount of usable scintillation light detected by the photomultiplier tubes (PMTs) which are coupled to the BGO block. The statistical noise in these photon signals is the crucial factor limiting the position resolution capability of the detectors. In the modified blocks, a portion of the available light was used for measuring the DOI and was, in the process, subtracted from that available for the normal transverse position measurement. As a result, the usual energy and position resolutions of the detector were somewhat degraded. This degradation, which showed up as a loss of resolution at the centre of the FOV, is an essential trade-off for an improvement in offcentre resolution by this method. To measure the amount of lost resolution was an essential goal of this work.

The hrplus block detector is shown schematically in Fig. 1. The BGO block is segmented into 64 small crystals which are optically separated from each other by narrow saw cuts filled with opaque material. In the unmodified blocks, the filling material is a white reflector, designed to direct as much of the scintillation light as possible to the back face where the optical photons are detected by an array of 4 PMTs. In the modified blocks, a small portion of the white reflector was replaced by inserting black modeling clay between the side walls of each small crys$\mathrm{tal}$, as indicated by the heavy black lines at the top end of each crystal in Fig. 1. The addition of the black absorbing material introduces a depth dependence to the number of 
jections, normalized counts as a function of two lengths and $t w 0$ angles [8]. were acquired in a technique similar 1.0 that used in [5]. Measuring the radial width of a point source peak in the projections was accomplished simply by rotating only one detector.

To assess the essential effects of uncorrected DOI broadening in the unmodified hrplus detectors, the projected width of the central point source $(R s=0)$ was compared with the width of the same source measured off-centre ( $R s=75 \mathrm{~mm}$ ). Then the blocks were modified and the same measurements repeated. The success of the correction technique was finally assessed by measuring the degree to which the resolution at $R s=75 \mathrm{~mm}$ could be restored to be the same as the central resolution measured before modification.

Calibration data, acquired beforehand, were used to correct the $4 \mathrm{D}$ projection arrays for transverse distortion, energy thresholding, crystal-to-crystal efficiency, and finally, depth distortion.

\section{A. Block Modification Technique}

The hrplus blocks were modified in two steps. First a shallow fill. $2.5 \pm 0.5 \mathrm{~mm}$ deep, was done, and the depth sensitivity [10] measured using the fanbeam technique described in the next section. Next, a $5 \mathrm{~mm}$ deep filling

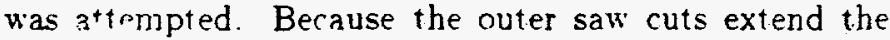
full io $\mathrm{mm}$ depth (see Fig. 1), the outer rows of crystals were vulnerable to separating from the central $6 \times 6$ crystals of the block. The first attempt to push the black clay $5 \mathrm{~mm}$ deep into the saw cuts damaged the outer rows of crystals, which broke loose from the optical glue holding them to the faces of the PMTs. This damage degraded the light collection from the outer rows, but did not affect the functioning of the inner $6 \times 6$ crystals.

Successful filling of the second hrplus block was accomplished by cutting the metal can away from the front $8 \mathrm{~mm}$ of the block. Prior to filling the central part of the block, modeling clay was pushed into the saw cuts from the sides of the block, which avoided fracturing the outer rows.

\section{B. Transverse Position Calibration}

2D position calibration maps were measured and used to remove transvers $\epsilon$ position distortion. Fig. 3 (top) shows the position map of an unmodified hrplus block, determined with $511 \mathrm{keV}$ gamma rays. A flood source was formed by placing a point source at a large distance from the detectors. To minimize possible counting rate effects, the pulse current from the PMTs was adjusted to be identical in the calibration runs as in the final projection measurements, i.e. the equivalent of $7000 / \mathrm{s} 511 \mathrm{keV}$ gamma rays detected in each block. New maps were measured each time the blocks were modified or when the PMT voltages were changed to adjust the gains. Event-by-event data from the flood measurement was read by a histogramming program and formed into a $128 \times 1282 \mathrm{D}$ array of counts vs $X_{m}$ and $Y_{m}$, where $X_{m}$ and $Y_{m}$ were the horizontal and vertical transverse positions calculated from the ratios of PMT pulse heights [7]. This $2 \mathrm{D}$ array showed peaks corresponding to each
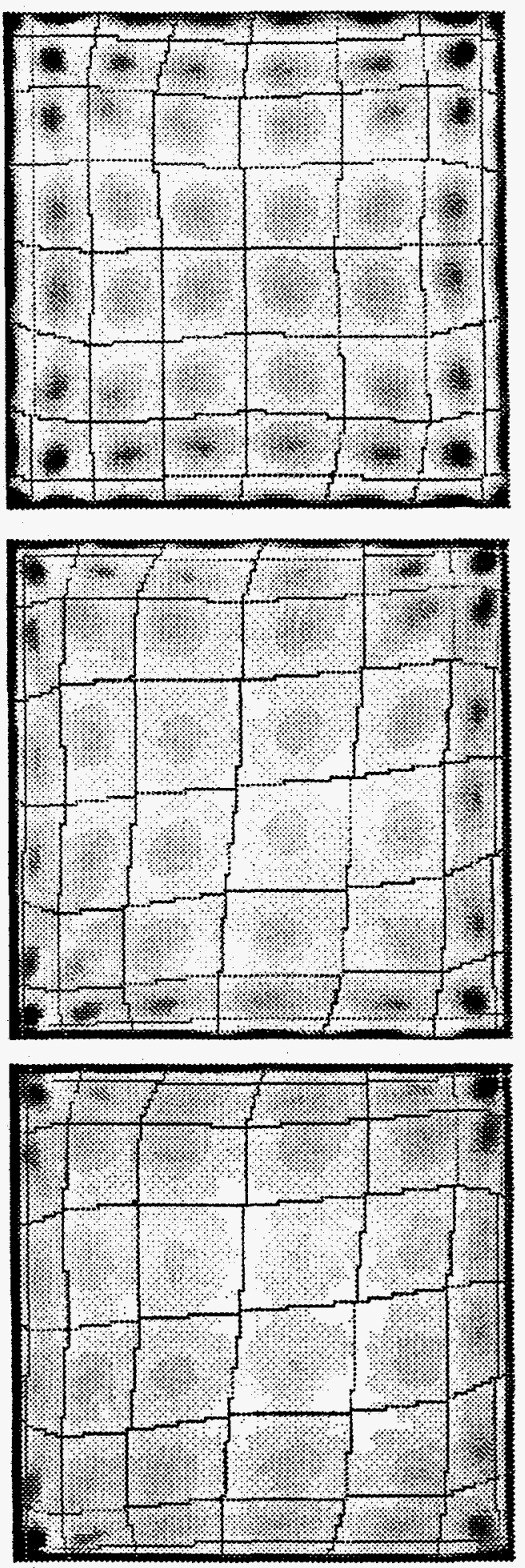

Fig. 3. Typical crystal position maps (solid lines) overlaying 2D flood calibration data, used to determine maps. Top=unmodified hrplus, map and data from ${ }^{68} \mathrm{Ge}$. Middle=modified hplus, map and data from ${ }^{60} \mathrm{Co}$. Bottom=modified hrplus, map from ${ }^{60} \mathrm{Co}$ and data from ${ }^{68} \mathrm{Ge}$.

of the to 64 crystals in the block. The position of each peak was located by a local-maximum search routine. The $X_{m}$ and $Y_{m}$ coordinates of the intersection points of the cuts segmenting the block were calculated by averaging the $X_{m}$ and $Y_{m}$ coordinates of the maxima in 


\section{DISCLAIMER}

Portions of this document may be illegible in electronic image products. Images are produced from the best available original document. 


\section{DISCLAIMER}

This report was prepared as an account of work sponsored by an agency of the United States Government. Neither the United States Government nor any agency thereof, nor any of their employees, makes any warranty, express or implied, or assumes any legal liability or responsibility for the accuracy, completeness, or usefulness of any information, apparatus, product, or process disclosed, or represents that its use would not infringe privately owned rights. Reference herein to any specific commercial product, process, or service by trade name, trademark, manufacturer, or otherwise does not necessarily constitute or imply its endorsement, recommendation, or favoring by the United States Government or any agency thereof. The views and opinions of authors expressed herein do not necessarily state or reflect those of the United States Government or any agency thereof. 


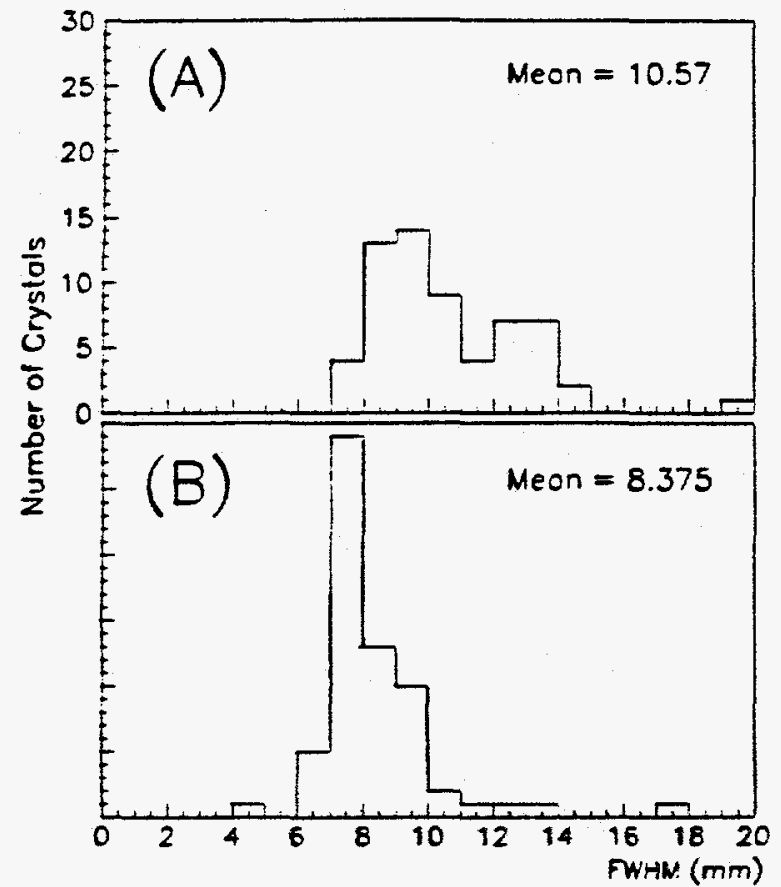

Fig. 6. The distribution of measured FWHM depth resolution among the 64 crystals of the modified hrplus blocks: (A) after the $2.5 \mathrm{~mm}$ fill and (B) after the final $5 \mathrm{~mm}$ fill.

64 crystals measured with the fanbeam at $Z=12 \mathrm{~mm}$ depth. The final (i.e. $5 \mathrm{~mm}$ ) fill improved the average resolution at this depth from 10.5 to $8.3 \mathrm{~mm}$ FWHM. At a single depth, such as that shown, depth resolution varied from crystal to crystal according to photon statistics, being worst in the corner crystals, where the light collection was the poorest. Photon statistics also dominated the variation of depth resolution with depth in each crystal; depth resolution was best at the front face, where the the absolute statistical uncertainty of the photopeak pulse-height was the smallest.

\section{Projections and Lines-of-Response}

Projections were formed by binning events into arrays, indexed by lines-of-response (LORs). Fig. 2 shows a horizontal section of the apparatus with a typical event represented by its LOR. Without DOI correction, each such LOR was determined by the measured transverse positions of the two gamma rays detected by the two detectors operated in coincidence. For each event, previously acquired 2D maps were referenced by the $X_{m}, Y_{m}$ words from the list-mode data file to fetch the column and row addresses, $0-7$, of each gamma ray. Each end of the LOR was defined horizontally by the column address and vertically by the row address of a crystal. From pairs of horizontal and vertical addresses, 4 LOR descriptors were formed to record each event. The sum of the two horizontal indices is called $L_{x}$, the radial position of the LOR. The difference of the two horizontal indices is called $\phi$, the azimuthal angle of the LOR. The sum of the two vertical indices is called slice, the axial position of the LOR. And finally, the difference of the two vertical indices is called $\theta$, the polar angle of the
LOR [8].

Since, in this geometry, DOI degrades only the radial component of resolution, not the slice component, we analyzed only a one-dimensional section of the $4 \mathrm{D}$ projection, namely counts vs $L_{x}$ for fixed values of slice, $\theta$, and $\phi$. Following [5], we integrated over a small range, i.e. $1.5^{\circ}$, in the angles about the direction perpendicular to $L_{x}$, which amounted to three bins in the units of the differences of crystal addresses. Three bins in slice were also summed over, to minimize the partial volume effect.

\section{E. Crystal Efficiency, Normalization, and Threshold}

The counts in each $4 \mathrm{D}$ projection bin were normalized to account for differences in the detection efficiencies of the two crystals defining the LOR. Individual crystal efficiencies were measured in separate data acquisition runs, using a ${ }^{68} \mathrm{Ge}$ point source located at a large enough distance from the detector to flood it with a uniform flux of $511 \mathrm{keV}$ gamma rays. To minimize scattering effects, an identical opposite trigger counter was operated in coincidence with the detector being calibrated, but at a smaller distance to the source so that only the uniform-efficiency central region of the trigger counter was effectively in coincidence with the detector being calibrated. The number of counts in the pulse-height spectrum of each crystal above a threshold channel was integrated and scaled to represent the crystal's fraction of the flood counts detected in the entire block. In the hrplus blocks the threshold channel numbers were chosen as a fraction $200 / 511$ or $350 / 511$ of the flood spectrum's photopeak channel number. In the modified blocks, threshold channels were similarly chosen using depth-separated pulse-height spectra, measured with a fanbeam located at $\mathrm{Z}=2 \mathrm{~mm}$ from the front face, as described in Section $\mathrm{C}$ above.

Detection efficiency also depends on the angle that the LOR makes with the normal to the block face. For projections acquired with the central source (Rs=0), this angle was nearly zero, while for projections acquired with the source at $\mathrm{Rs}=75 \mathrm{~mm}$, the angle to the normal averaged about $16^{\circ}$. To account for efficiency variation with this angle, efficiency calibrations were done separately at $0^{\circ}$ and $16^{\circ}$ and were used to analyze the $R s=0$ and $R s=75 \mathrm{~mm}$ projection data, respectively.

The flood source normalizations, described above, were applied event-by-event in forming the normalized projections. A table of threshold pulse-heights, along with the relative crystal efficiencies, was referenced for each detected gamma ray. If both gamma rays of an event were found to be above their respective thresholds, a normalized increment for the event was calculated as the reciprocal of the product of the two tabulated crystal efficiencies. This normalized increment was added to the projection to record the event. This procedure accounted for all crystal efficiency variations between the LOR values measured at a single angular position setting of the movable detector.

Other normalization effects, including solid-angle variation with the distance between the detectors, variation in run duration, and computer dead-time losses, were calculated and accounted for by a separate relative normal- 


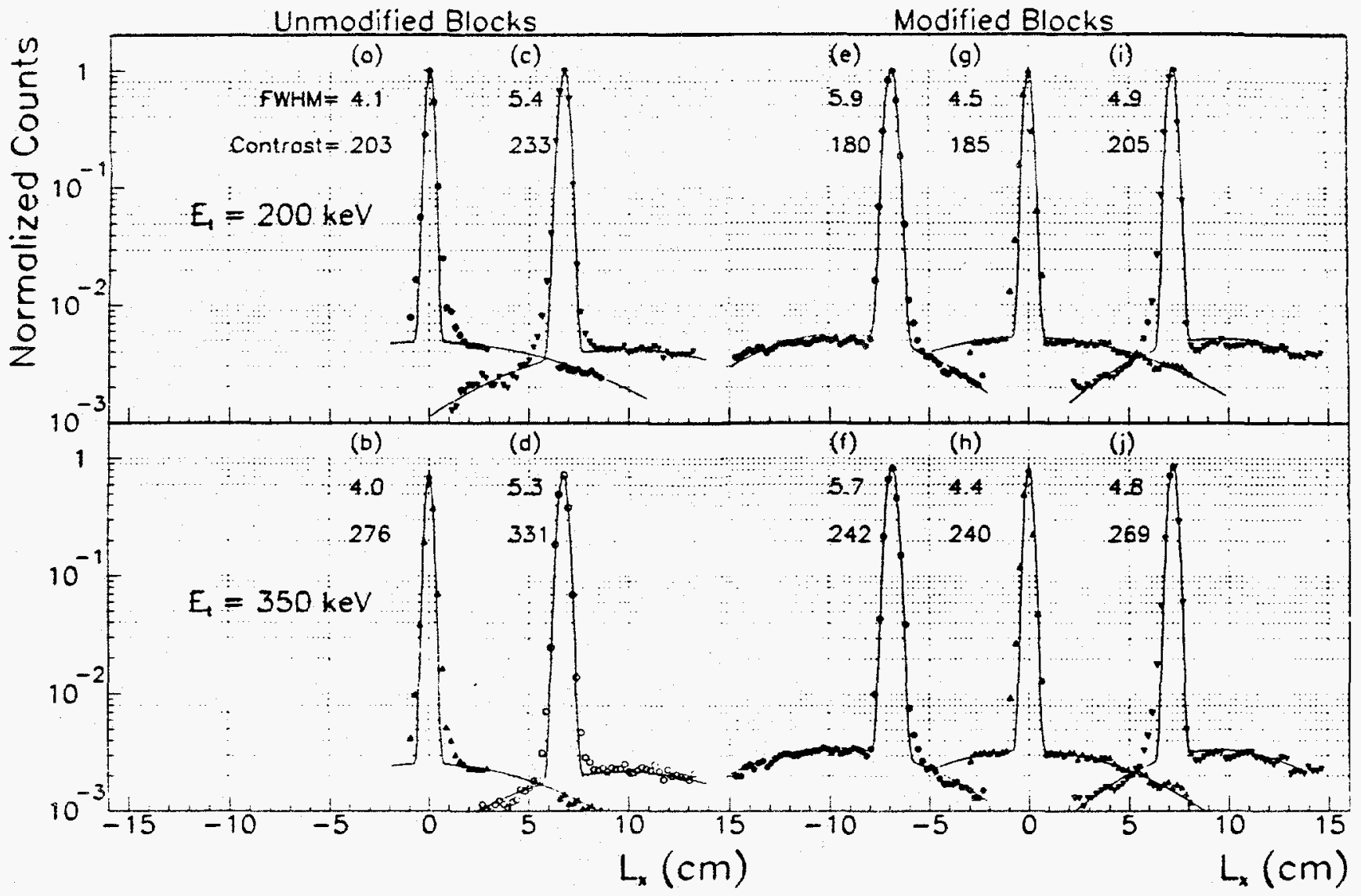

Fig. 9. Radial point-source projections measured with energy threshold $200 \mathrm{keV}$ (upper panel) and $350 \mathrm{keV}$ (lower panel). Source, detector configurations were, from left to right, (a-b) central in FOV, unmodified blocks; (c-d) $75 \mathrm{~mm}$ off-centre radially, unmodified; (e-f) off-centre, uncorrected; $(\mathrm{g}-\mathrm{h})$ central, uncorrected; and (i-j) off-centre, corrected by the new method. FWHM $=$ the width of the fitted Gaussian (solid curve). Contrast $=$ the ratio of the unscattered to scattered peak amplitudes.

of these peaks shift with varying PMT gains by amounts which vary from crystal to crystal, depending on the 4 independent PMT gain changes and modulated by the light sharing among the PMTs. Since light sharing doesn't depend on PMT gains, it follows that the relative shift in the peaks of the fiood spectra is the same as that for peaks of the fanbeam spectra. Hence measuring the fractional shift in the peaks of the flood spectra provided scaling factors for shifting the fanbeam peaks.

To validate this new method of depth calibration, the fanbeam peak positions at $1600 \mathrm{~V}$ were scaled up by the ratio of the maximum pulse-heights determined from the flood calibration shown in Fig. 7 and are compared to measured $1700 \mathrm{~V}$ fanbeam depth responses in Fig. 8. The disagreements between the flood and fanbeam curves in Fig. 8 were judged to be small enough to be safely ignored, considering that the gain shifts produced by the test $100 \mathrm{~V}$ change are much larger than those expected in normal camera operation. The remaining discrepancies appear to be due to statistics and could therefore have been reduced by smoothing the spectra prior to locating the peaks. An obvious example of such a statistical error is in the third down and third from the left panel in Fig. 7, in which the $1700 \mathrm{~V}$ peak-search failed due to a visible statistical anomaly.

\section{RESULtS}

Fig. 9 shows the measured projections of point sources with the unmodified (a-d) and modified (e-j) hrplus block detectors. The top panel was acquired with a "low" energy threshold of $200 \mathrm{keV}$ and the bottom panel with a "high" threshold of $350 \mathrm{keV}$. Only the non-overlapping central seven points of ea detector position are included in the projections; LOR values measured with the edge row's of crystals were omitted because of the more reliable efficiency calibration of the interior crystals. In the central projections, near $\mathrm{L}_{x}=0$, the $\mathrm{L}_{x}$ samples are plotted at $1 / 2$ crystal intervals, $2.25 \mathrm{~mm}$. Off-centre, the $\mathrm{L}_{x}$ samples are plotted at intervals foreshortened by the cosine of the angle between the block normals, i.e. at $2.25 \times \cos \left(16^{\circ}\right)=2.16 \mathrm{~mm}$. The sum of two fitted Gaussians is shown with each projection by a solid line, which passes through the fitted points. Gaussians were fitted separately to five points in the narrow peaks, which measures point source spatial resolutions, and to the underlying backgrounds, which measures the scatter contamination from the $20 \mathrm{~cm}$ Lucite cylinder. The systematic uncertainty in the fitted FWHM values, due to the departure of the measured projections from true Gaussian shape, is estimated to be $\pm 0.1 \mathrm{~mm}$. The fitted FWHM values are $0.1 \mathrm{~mm}$ better in the higherthreshold projections than in their lower threshold coun- 
increased slope.

A promising method of getting more light out of the crystals is to replace the $\mathrm{BGO}$ by a brighter scintillator, such as LSO [15]. The rapid decrease in the bulk price of lutetium oxide [16], and the recent production of large, clear crystals of LSO [17], make such a DOI-correcting LSO block detector a real possibility for the next generation PET cameras.

Due to limited time and budget, this study was not extended to large distances from the centre of the FOV or to large scattering phantoms. Since our objective is to eventually utilize the entire ring diameter for imaging, additional measurements are needed in the future to investigate the performance of detectors and $\mathrm{DOl}$ correction algorithm for sources at large radii. For torso imaging, DOI correction in larger scattering phantoms should also be assessed.

\section{REFERENCES}

[1] S.E. Derenzo, W.W. Moses, H.G. Jackson, B.T. Turko, J.L. Cahoon, A.B. Geyer, and T. Vuletich, "Initial characterization of a position sensitive photodiode/BGO detector for PET", IEEE Trans. NS-36, 1084-1089 (1989).

[2] J.G. Rogers, "A method for correcting the Depth-ofinteraction blurring in PET cameras", IEEE Trans. MI-14, 146-150 (1995)

[3] M.E. Casey, R. Nutt, T.D. Douglass, "Process for fabricating tuned light guide for photoelectrons", U:S. Patent \#4,750,972 (1988).

[4] M.E. Casey and R. Nutt, "A multicrystal two dimensional BGO detector system", JEEE .Trans. NS-33, 460-467 (1986).

[5] M.J. Lercher and $k$. Wienhard, "Scatter correction in 3-D PET", IEEE Trans. MI-13, 649-65T (1994).

[6] M. Dahlbom, L. Eriksson, and K. Wienhard, "Design study of future 3D PET systems", MIC Conference Record, Norfolk, VA, 1667-1671 (1995)

[7] J.G. Rogers, A.J. Taylor, M.F. Rahimi, R. Nutt, M. Andreaco, and C.W. Williams, "An improved multicrystal 2D BGO detector for PET", IEEE Trans. NS-39, 1063-1068 (1992).
[8] J.G. Rogers. R. Harrop, and P.E. Kinahan. "The theory of three-dimensional image reconstruction for PET", IEEE Trans. MI-6, 239-243 (1987).

[9] C. Moisan, J.G. Rogers, K.R. Buckley, T.J. Ruth, M.W. Stazyk, and G. Tsang, "Design studies of a depth encoding large aperture PET camera", IEEE Trans. NS-42, 1041-1050 (1995).

[10] J.G. Rogers, R. Nutt, M. Andreaco, and C.W. Williams, "Testing 144- and 256-crystal BGO block detectors", IEEE Trans. NS-40, 1001-1007 (1993).

[11] W.W. Moses, R.H. Huesman, and S.E. Derenzo, "A new algorithm for using DOI measurement information in PET data acquisition", J. Nucl. Med. Vol. 32. Abstract \#365 (1991).

[12] M.E. Casey, "An analysis of counting losses in positron emission tomography", Ph.D. Thesis, University of Tennessee, Knoxville, December, 1992.

[13] W.F. Jones, M.E. Casey, L.G. Byars, and S.G. Burgiss, "A VMEbus real time sorter design for positron emission tomography", IEEE Trans. NS-33, 601-604 (1986).

[14] C. Moisan, P. Tupper, J.G. Rogers and J.K. deJong, "A Monte Carlo study of the acceptance to scattered events of a depth encoding PET camera", IEEE 1995 Medical Imaging Conference Record and submitted to IEEE Trans. Nucl. Science.

[15] C.L. Melcher and J.S. Schweitzer, "Cerium-doped lutetium oxyorthosilicate: a fast, efficient new scintillator", IEEE Trans. NS-39, 502-505 (1992).

[16] W.W. Moses, S.E. Derenzo, A. Fyodorov, M. Korzhik, A. Gektin, B. Minkov, and V. Aslanov, "LuAlO3:Ce - A high density, high speed scintillator for gamma detection", IEEE Trans. NS-42, 275-279 (1995).

[17] M.J. Paulus, J.M. Rochelle, M.S. Andreaco, D.M. Binkley, "A low-noise, wide-band, CMOS chargesensitive preamplifier for use with APD/LSO PET detectors", poster NSS13-20, presented at 1995 IEEE Nuclear Science Symposium, San Francisco, CA, October 25, 1995. 